\title{
Overexpression of novel long intergenic non-coding RNA LINC02454 is associated with a poor prognosis in papillary thyroid cancer
}

\author{
JUAN TAN ${ }^{1,2^{*}}$, LING LIU $^{1 *}$, ZHIHUA ZUO ${ }^{1}$, BIN SONG $^{3}$, TINGTING CAI ${ }^{1}$, \\ DAFA DING ${ }^{1}$, YIBING LU ${ }^{1}$ and XIAOLONG YE ${ }^{1}$
}

${ }^{1}$ Department of Endocrinology, The Second Affiliated Hospital of Nanjing Medical University, Nanjing, Jiangsu 210011;
${ }^{2}$ Department of Gerontology, The Affiliated Huaian No. 1 People's Hospital of Nanjing Medical University,
Huai'an, Jiangsu 223300; ${ }^{3}$ Department of Endocrinology, Clinical Medical College of Yangzhou University,
Yangzhou, Jiangsu 225001, P.R. China

Received October 6, 2019; Accepted May 4, 2020

DOI: $10.3892 /$ or.2020.7712

\begin{abstract}
It has been revealed from microarray data analysis that long intergenic non-coding RNA 02454 (LINC02454) is highly expressed in papillary thyroid cancer (PTC). The aim of the present study was to explore the potential role of LINC02454 in the tumorigenesis of PTC. The mRNA expression levels of LINC02454 were assessed using data from The Cancer Genome Atlas (TCGA) and the GSE66783 cohort in thyroid cancer, and were validated using reverse transcription-quantitative PCR in 104 patients with PTC recruited in the present study. The association between the LINC02454 mRNA expression levels and the clinicopathological features of the 104 patients with PTC were also analyzed. Functional enrichment analyses were conducted on the differentially expressed genes in the high and low LINC02454 expression groups that were identified from the TCGA cohort. RNA interference, using short interfering (si)RNA against LINC02454, was used to investigate the role of LINC02454 in the biological functions of PTC cells in vitro. The expression level of LINC02454 was significantly increased in PTC tissues $(\mathrm{P}=0.0011)$ and was significantly associated with a larger tumor size, T stage, an advanced TNM stage and an increased lymph node metastasis $(\mathrm{P}<0.05)$, which was consistent with that in the
\end{abstract}

Correspondence to: Dr Yibing Lu or Xiaolong Ye, Department of Endocrinology, The Second Affiliated Hospital of Nanjing Medical University, 121 Jiangjiayuan Road, Nanjing, Jiangsu 210011, P.R. China

E-mail: luyibing2007@163.com

E-mail: xiaolongye2004@126.com

*Contributed equally

Key words: LINC02454, papillary thyroid cancer, proliferation, apoptosis
TCGA and GSE66783 cohort. High expression levels of LINC02454 were observed in patients with PTC that also had BRAF mutations $(\mathrm{P}<0.001)$, and were significantly associated with a poorer disease-free survival in the TCGA cohort $(\mathrm{P}<0.05)$. Functional enrichment analysis indicated that LINC02454-related genes were significantly enriched in Gene Ontology terms, such as 'positive regulation of cell proliferation', 'positive regulation of cell division' and 'cell adhesion', and the following Kyoto Encyclopedia of Genes and Genomes pathways: 'Pathways in cancer' 'proteoglycans in cancer' and 'ECM-receptor interaction'. In vitro, the knockdown of LINC02454 markedly arrested the cells in the $G_{0} / G_{1}$ phase of the cell cycle, and also led to an overall increase in apoptosis, as well as to an unexpected decrease in cell proliferation. LINC02454 may thus potentially function as an oncogene, which inhibits the apoptosis and enhances proliferation of PTC cells. Thus, as suggested by the findings of the present study, LINC02454 may be used as a diagnostic and prognostic biomarker for PTC in the future.

\section{Introduction}

Over the past 4 decades, there has been a sharp increase in the number of cases of thyroid cancer, and it is recognized as the most frequent endocrine malignancy worldwide $(1,2)$. In the USA, thyroid cancer is the fifth most common type of cancer among women, and the most frequently diagnosed malignancy in individuals aged $<30$ years in China $(1,3)$. In addition, in 2016 , it was reported that 64,300 individuals were diagnosed with thyroid cancer in the USA (4). The incidence rate of thyroid cancer in China is 6.6 per 100,000 individuals (5). Papillary thyroid cancer (PTC) accounts for $\sim 80 \%$ of all cases of thyroid cancer (6). Patients with PTC have a more favorable prognosis, which is attributed to improvements in radiotherapy, endocrine replacement therapy and complete thyroidectomy (7). However, $>25 \%$ of patients experience recurrence during long-term follow-up (8). Therefore, the identification of novel diagnostic and prognostic biomarkers is crucial for patients with PTC. 
It has been demonstrated that PTC is associated with mutations, such as point mutations in B-Raf proto-oncogene, serine/threonine kinase $(B R A F)$ and RAS GTPase $(R A S)$ or gene rearrangements in either ret proto-oncogene $(R E T)$, neurotrophic receptor tyrosine kinase 1 (NTRK1) and anaplastic lymphoma kinase $(A L K)$ (9-12). In addition, long non-coding RNAs (IncRNAs), have been found to be important contributors to the oncogenesis of PTC $(13,14)$. IncRNAs do not possess protein-coding capacities and usually consist of $>200$ nucleotides, and can be categorized into antisense, sense, bidirectional, intronic and intergenic lncRNAs according to their genomic context (15). IncRNAs can regulate gene expression and are involved in numerous biological processes, such as cell invasion and migration, apoptosis and proliferation, cell cycle and cell proliferation (13), and were initially observed to be aberrantly expressed during tumorigenesis (16). For example, several lncRNAs function as tumor promoters in PTC, such as nuclear paraspeckle assembly transcript 1 (NEAT1) (17), LOC100507661 (18), colon cancer-associated transcript 1 (CCAT1) (19) and AFAP1 antisense RNA 1 (AFAP1-AS1) (20), while other lncRNAs function as tumor suppressors for example, papillary thyroid cancer susceptibility candidate 3 (PTCSC3) (21), maternally expressed 3 (MEG3) (22) and long intergenic non-coding RNA 271 (LINC00271) (23). In addition, H19 has been reported to function as both a suppressor and promoter of tumorigenesis in different PTC cell lines $(24,25)$. Despite recent progress in the understanding of the functions of IncRNAs, their functional roles remain elusive. In particular, the involvement of lncRNAs in thyroid tumorigenesis has not yet been fully explored.

The present study aimed to explore differentially expressed lncRNAs in PTC tissue and paired adjacent normal tissue samples using a lncRNA microarray and to identify the functional role of LINC02454 in PTC oncogenesis.

\section{Materials and methods}

Tissue acquisition. A total of 104 thyroid cancer tissue and paired adjacent normal samples were obtained from patients with thyroid cancer who received a thyroidectomy at the Second Affiliated Hospital of Nanjing Medical University between 2015 and 2017. Each specimen was immediately flash-frozen in liquid nitrogen before storage at $-80^{\circ} \mathrm{C}$ until further experimentation. The following criteria were used for patient selection: No previous treatment and a diagnosis of PTC confirmed by histopathological examination. The $8^{\text {th }}$ edition of the American Joint Committee on Cancer classification system was used for tumor-node-metastasis (TNM) staging (26). The study protocols were reviewed and approved by the Ethics Committee of the Second Affiliated Hospital of Nanjing Medical University (no. 2014 KY no. 054). All patients provided written informed consent prior to participation.

Hematoxylin and eosin staining. The pathological tissues were obtained by surgery, and fixed with $10 \%$ formaldehyde and then embedded in paraffin and sliced at a thickness of $4 \mu \mathrm{m}$. For hematoxylin and eosin (H\&E) staining, paraffin-embedded thyroid tissue sections were deparaffinized with xylene for $5 \mathrm{~min}$ (this process was repeated once), $100 \%$ ethanol for $5 \mathrm{~min}$,
$90 \%$ ethanol for $2 \mathrm{~min}, 80 \%$ ethanol for $2 \mathrm{~min}, 70 \%$ ethanol for $2 \mathrm{~min}$, and washed with distilled water for $2 \mathrm{~min}$. The sections (4- $\mu \mathrm{m}$-thick) were stained with hematoxylin stain (Beyotime Institute of Biotechnology, Inc.) for $5 \mathrm{~min}$ and washed with distilled water for $10 \mathrm{~min}$ at room temperature. The sections were differentiated with acid alcohol slow differentiation solution (Beyotime Institute of Biotechnology, Inc.) for $30 \mathrm{sec}$ and soaked in distilled water for $15 \mathrm{~min}$. The sections were stained with eosin stain (Beyotime Institute of Biotechnology, Inc.) for $2 \mathrm{~min}$ at room temperature. Slides were viewed with a Nikon Eclipse 80i microscope equipped with a digital camera (DS-Ri1; Nikon Corp.).

Microarray profiling. RNA was isolated from 4 thyroid cancer tissues and 4 paired adjacent normal tissues and used for integrated lncRNA/mRNA microarray analysis (CapitalBio Technology, Inc.). RNA preparation and hybridization of the arrays were performed with minor alterations to the manufacturer's protocols. Firstly, mRNA was purified to remove ribosomal RNA with the mRNA-ONLY Eukaryotic mRNA Isolation kit (Epicentre; Illumina, Inc.). Fluorescent cRNAs were produced using amplification and transcription of the whole transcriptome, to avoid 3'bias. The arrays were subsequently processed using an Agilent scanner and the images were analyzed using the Agilent Feature Extraction software (version 11.0.1.1), while the GeneSpring GX v12.0 software package was used to normalize quantiles and for subsequent data processing (all Agilent Technologies, Inc.). IncRNAs and mRNAs that possessed tags of present or marginal (All Targets Value) in the 4 samples following quartile normalization of the raw data were subjected to additional analyses. Hierarchical clustering was used to illustrate patterns of IncRNA and mRNA expression.

Cell lines and cell culture. The 2 PTC cell lines used in the present study (TPC1 and IHH4) were a gift from Dr Chao Liu of Nanjing University of Chinese Medicine. The cells were cultured with RPMI-1640 medium (Invitrogen; Thermo Fisher Scientific, Inc.) supplemented with $1 \%$ penicillin/streptomycin and $10 \%$ fetal bovine serum (Gibco; Thermo Fisher Scientific, Inc) at $37^{\circ} \mathrm{C}$ in a humidified incubator with $5 \% \mathrm{CO}_{2}$.

RNA isolation, cDNA synthesis and reverse transcription-quantitative PCR (RT-qPCR). RNA was extracted from cell lines, tumor tissues, and adjacent normal tissues using TRIzol ${ }^{\circledR}$ reagent (Invitrogen; Thermo Fisher Scientific, Inc.). The RevertAid First Strand cDNA synthesis kit (Thermo Fisher Scientific, Inc.) was used to synthesize cDNA according to the manufacturer's instructions. An ABI 7500 Real-Time PCR system was used with SYBR-Green Real-Time PCR master mix (Toyobo Life Science) to determine the mRNA expression levels of LINC02454 using the recommended protocols. The PCR reactions were repeated for 40 cycles under the following conditions: $95^{\circ} \mathrm{C}$ for $15 \mathrm{sec}$, $60^{\circ} \mathrm{C}$ for $15 \mathrm{sec}$ and $72^{\circ} \mathrm{C}$ for $45 \mathrm{sec}$. Relative expression levels were determined using the $\Delta \mathrm{Ct}$ method, and GAPDH expression was used as the internal control. The following primers were used, which were synthesized by Invitrogen (Thermo Fisher Scientific, Inc.): LINC02454 forward, 5'-GCTTGAACATCG TCCTCCTC-3' and reverse, 5'-TGTTCTCTGTGGGAATGC AA-3'; GAPDH forward, 5'-GAAGGTGAAGGTCGGAGTC-3' and reverse, 5'-GAAGATGGTGATGGGATTTC-3'. 
The Cancer Genome Atlas (TCGA) analysis. RNA sequencing data for 502 thyroid tumor samples and 58 normal tissue samples were retrieved from TCGA (https://portal.gdc.cancer. gov/). Clinical information, such as lymph node count, TNM stage and pathological stage was also downloaded. Information regarding the BRAF mutation status, as well as the status and time of disease-free survival were collected from the cBioPortal for Cancer Genomics (http://www.cbioportal.org/). A total of 484 patients had complete survival information. The limma package (27) of R was used to identify differentially expressed lncRNAs between PTC and adjacent normal samples. $\mathrm{P}<0.05$ and a fold change (FC) $>2$ were selected as cut-off values. The expression levels of LINC02254 was also investigated for an association with different clinical traits. For association analysis, only the tumor samples were used. Kaplan-Meier survival analysis was performed to examine the potential impact of the expression level of LINC02454 on the prognostic survival of patients with PTC. Receiver operating characteristic (ROC) curve analysis was used to examine the powers of the levels of LINC02454 expression for disease-free survival diagnosis. The Youden index was set as the cut-off point to estimate sensitivity, specificity and positive diagnostic likelihood ratio. The high and low levels of LINC02454 expression groups were set up based on the Youden index. Kaplan-Meier survival analyses were performed to examine the potential effects of the expression level of LINC02454 on the prognostic survival of the 484 patients with PTC. Additionally, the association of the disease-free survival time of the patients with PTC with clinical traits was also analyzed.

Verification of LINC02254 expression in PTC using the GEO dataset. The IncRNA expression profiles of 5 pairs of PTC and paired adjacent non-cancerous thyroid tissue samples were downloaded from GEO (Accession no. GSE66783). The probe CUST_4297_PI429285431 corresponds to a transcript of the LINC02454, which is ENST00000539116.1.

Identification of LINC02454-associated genes and protein-protein interaction (PPI) network. The 502 PTC samples downloaded from TCGA were divided into 2 groups based on the median expression level of LINC02454. The differentially expressed mRNAs between the low and high LINC02454 expression groups were identified using the limma package in $\mathrm{R}(27)$. An adjusted $\mathrm{P}$-value (adj. P) $<0.01$ and $\mid \log \mathrm{FCl}>1$ were used as the cut-off values. The differentially expressed genes were determined to be LINC02454-associated genes and subsequently used for PPI analysis. The Search Tool for the Retrieval of Interacting Genes/Proteins (STRING) database (http://string-db.org/) contains PPIs (28), and was used to construct a PPI network of LINC02454-associated genes. The network was subsequently visualized using Cytoscape (29).

Functional enrichment analysis. The Gene Ontology (GO) (http://www.geneontology.org) database consists of 3 categories: Molecular function (MF), cellular component (CC) and biological process (BP) (30). Systemic, chemical and genomic information was obtained from the KEGG (http://www. genome.ad.jp/kegg/) database (31). Significantly enriched GO terms and KEGG pathways were identified using the Database for Annotation, Visualization and Integrated Discovery online tool (https://david.ncifcrf.gov/) with a threshold of $\mathrm{P}<0.05$ (32).
Small interfering (si)RNA transfection. LINC02454 siRNA (si-LINC02454) and a negative control (si-NC) were purchased from Shanghai GenePharma Co., Ltd. The siRNA sequences are presented in Table I. Lipofectamine ${ }^{\circledR} 2000$ (Invitrogen; Thermo Fisher Scientific, Inc.) was used to transfect both si-LINC02454 and si-NC into the TPC1 cells, which were incubated in 6-well plates. The TPC1 cells were grown to 50-70\% confluency and transfected with $100 \mathrm{nmol} / \mathrm{l}$ si-LINC02454 or si-NC following the manufacturer's protocols. The knockdown efficiency of si-LINC02454 was determined using RT-qPCR at $48 \mathrm{~h}$ following transfection.

Cell viability assay. The Cell Counting kit-8 (CCK-8; Dojindo Molecular Technologies, Inc.) assay was used to determine the rate of cellular viability. First, TPC1 cells (1,000 cells/well) were seeded into 96-well plates and transfected with siRNA. Following transfection for 24, 48, 72 and 96 h, $10 \mu \mathrm{l} \mathrm{CCK}-8$ reagent were added to each well. The cells were incubated for a further 2-4 h before the absorbance was measured at $450 \mathrm{~nm}$. Each assay was performed 3 times.

Colony formation assay. Transfected TPC1 cells (500 cells/well) were seeded in 6-well plates to evaluate monolayer colony formation. The cells were cultured were cultured with RPMI-1640 medium (Invitrogen; Thermo Fisher Scientific, Inc.) at $37^{\circ} \mathrm{C}$ for 6 days, and the culture medium was changed at regular time intervals. After 6 days, the adherent cells were washed twice with PBS and fixed with $4 \%$ paraformaldehyde for $30 \mathrm{~min}$ at room temperature. The colonies were stained for 15 min with $0.1 \%$ crystal violet (Chengdu Kelong Chemical Co., Ltd.) at room temperature before being rinsed with water and air-dried. Light microscopy was used to count the cell colonies ( $>50$ cells per colony). Each experiment was repeated 3 times.

Cell cycle and cell apoptosis assays. To determine the proportion of cells in each phase of the cell cycle, the TPC1 cells were fixed overnight at $4^{\circ} \mathrm{C}$ with cold $70 \%$ ethanol for $48 \mathrm{~h}$ following transfection with siRNA. A total of $100 \mu$ l RNase A reagent (Nanjing KeyGen Biotech, Co., Ltd.) was used to the treat cells prior to incubation at $37^{\circ} \mathrm{C}$ for $30 \mathrm{~min}$. Lastly, $400 \mu \mathrm{l}$ propidium iodide reagent (Nanjing KeyGen Biotech, Co., Ltd.) was used to stain the cells. DNA content was measured at $488 \mathrm{~nm}$ using a FACSCanto II flow cytometer (Becton-Dickinson and Company). For cell apoptosis analysis, TPC1 cells transfected with si-NC or si-LINC02454 were harvested $48 \mathrm{~h}$ following transfection, washed twice and stained with an Annexin V/PI apoptosis detection kit (Nanjing KeyGen Biotech, Co., Ltd.). Flow cytometry (FACSCanto II; BD Biosciences) was used to analyze apoptotic cells in early and late apoptosis. Each experiment was repeated 3 times.

Statistical analysis. SPSS v23.0 (IBM Corp.), GraphPad Prism v6.0 (GraphPad, Inc.) and R (version 3.5.1) (https://www.r-project.org/) were used to analyze all data. The one-sample Kolmogorov-Smirnov test was used for normality testing of continuous data, and data are presented as the means \pm standard deviation (SD) for normally distributed data or median (interquartile range, IQR) for skewed distribution. Error bars in the scatter plots and the bar graphs represent SD or IQR. 
Table I. siRNA sequences.

Si-RNA Primer sequences

si-LINC02454-1

si-LINC02454-2

si-LINC02454-3

siRNA-NC
Sense: 5'-GCUUCCAAGGUCAUGCCUUTT-3'

Antisense: 5'-AAGGCAUGACCUUGGAAGCTT-3'

Sense: 5'-GCCAGUUUCCUAAAUCAAATT-3'

Antisense:5'-UUUGAUUUAGGAAACUGGCTT-3'

Sense: 5'-GGCUUGGAGAUCCUCAUUUTT-3'

Antisense: 5'-AAAUGAGGAUCUCCAAGCCTT-3'

Sense: 5'-UUCUCCGAACGUGUCACGUTT-3'

Antisense: 5'-ACGUGACACGUUCGGAGAATT-3'

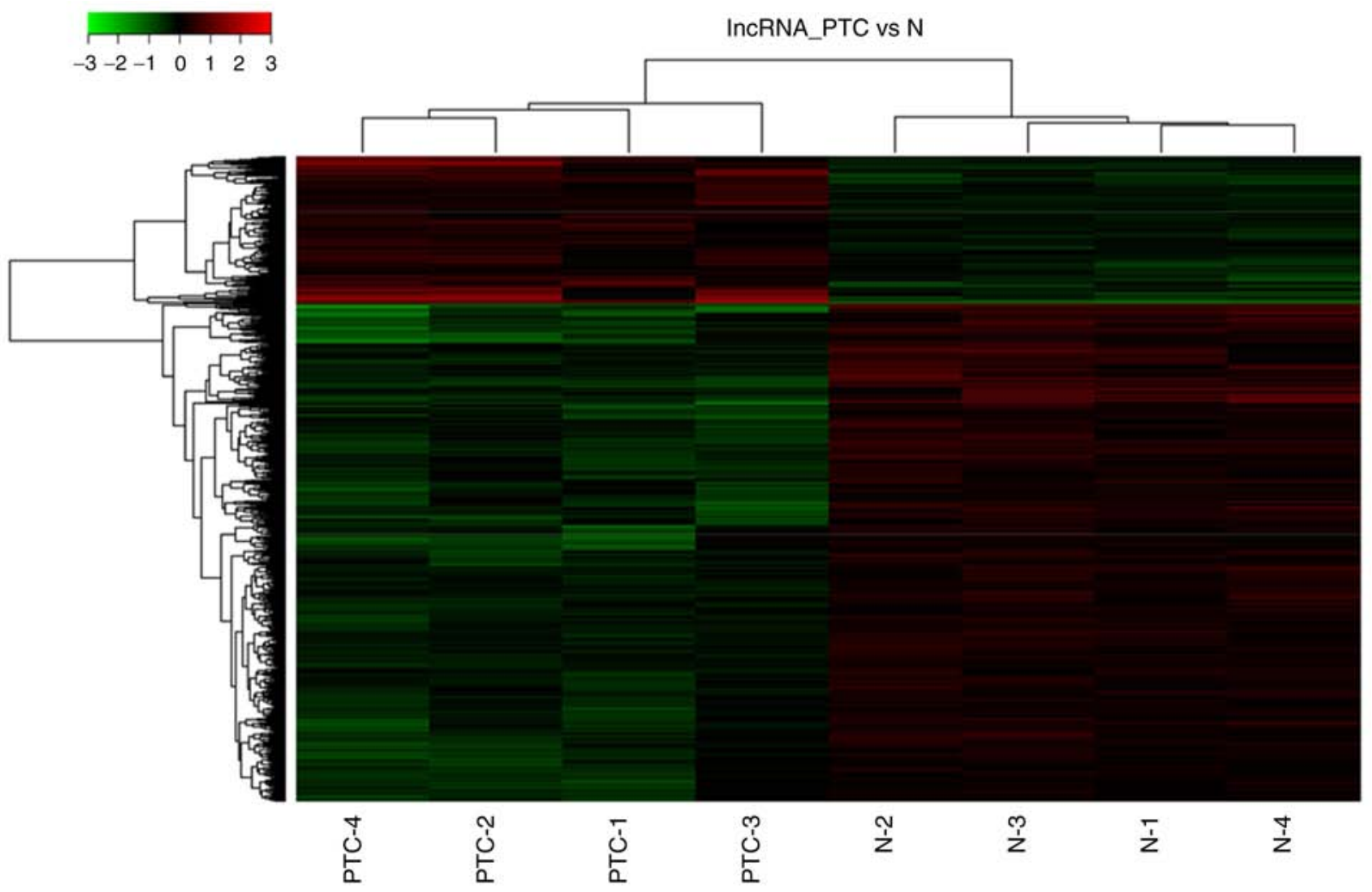

Figure 1. Heatmap of differentially expressed lncRNAs between papillary thyroid cancer tissues and matched adjacent non-cancerous thyroid tissues. Green color indicates a low expression, while red indicates a high expression. In the heatmap, columns represent samples and rows represent each lncRNA.

Significance between groups was determined using the Student's t-test and Mann-Whitney tests. The comparisons among $\geq 3$ groups were firstly performed by one-way ANOVA and Tukey's post hoc test if the variance between groups was comparable. When the data exhibited a skewed distribution, comparisons were performed using the non-parametric Kruskal-Wallis test with the Mann Whitney U post hoc test with Bonferroni's correction applied. Categorical data were reported as the count and percentage. The $\chi^{2}$ tests were used as appropriate for comparison of categorical data between groups. $\mathrm{P}<0.05$ (two-tailed) was considered to indicate a statistically significant difference. A ROC curve was generated by the package 'Optimal Cutpoints' (33) in R. Disease-free survival analysis was performed using the Kaplan-Meier survival curve and the log-rank test in the 'survival' (34) package of R (version 3.5.1).

\section{Results}

Overview of IncRNA expression profiles. Microarray expression profiling of 4 PTC tissues and paired normal tissues was performed to identify differentially expressed lncRNAs in PTC compared with normal tissues. A heatmap was generated to identify significantly differentially expressed lncRNAs (Fig. 1). The top 10 most significantly upregulated differentially expressed lncRNAs are presented in Table SI. In total, 6 of the top 10 lncRNAs (ENST00000417422.1, ENST00000457989.1, TCONS_00020761,ENST00000539116.1,ENST00000539653.1 and ENST00000563933.1) were also markedly upregulated in the PTC samples compared with the normal samples from the TCGA database (Fig. S1), which were selected for further analysis by RT-qPCR to confirm the expression levels in 
A

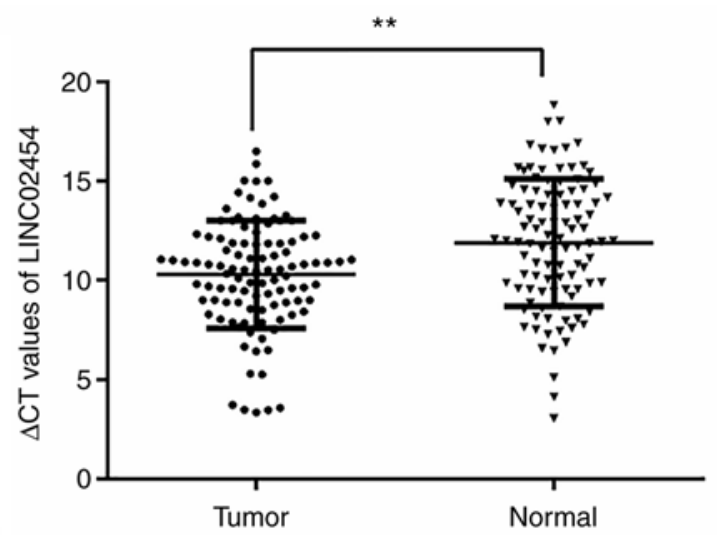

C

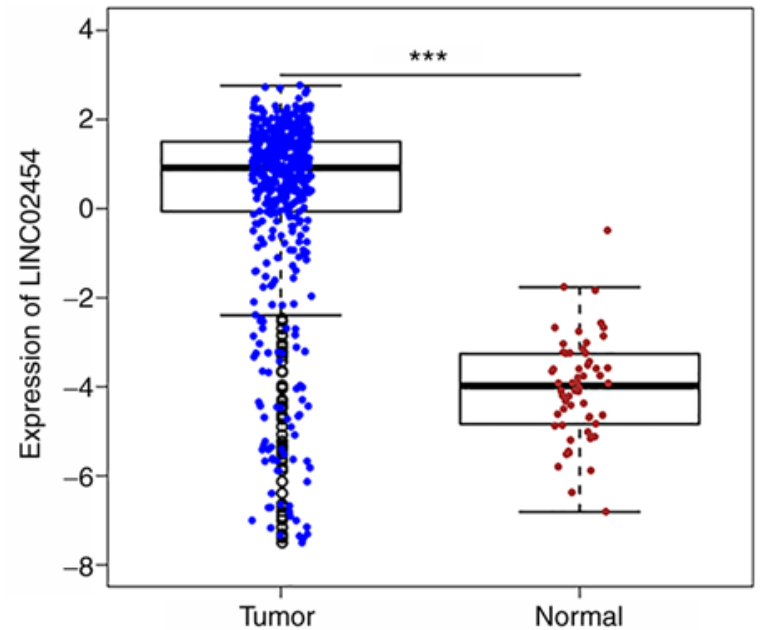

B

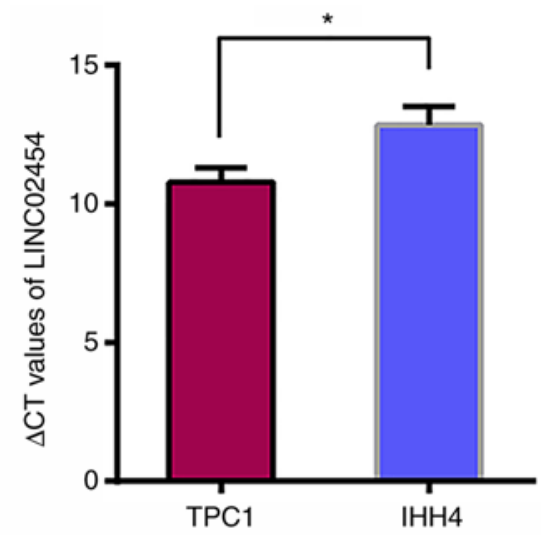

D

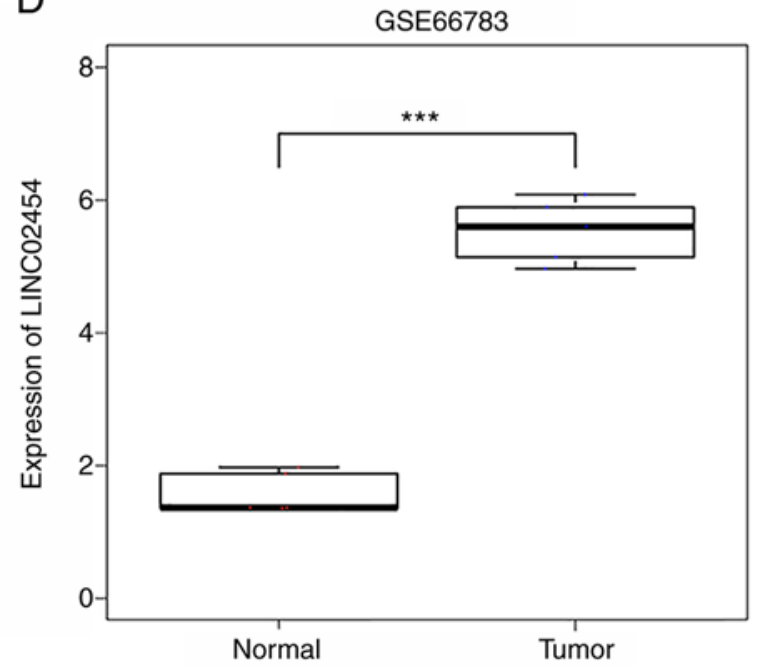

Figure 2. Expression of LINC02454 in PTC tissues and cell lines, and verification with TCGA and GEO data. (A) Relative expression of LINC02454 in PTC tissues and normal tissues $(\mathrm{P}=0.0011)$. (B) Relative expression of LINC02454 in TPC1 and IHH4 cells, which are both PTC cell lines $(\mathrm{P}=0.0136)$. LINC02454 expression was evaluated by RT-qPCR and normalized to GAPDH mRNA expression. The smaller the $\Delta \mathrm{Ct}$ value is, the higher expression. Data were analyzed using a paired t-test; ${ }^{\mathrm{P}}<0.05$ and ${ }^{* *} \mathrm{P}<0.01$. (C) Expression of LINC02454 in PTC tissues and adjacent normal tissues in TCGA data. Data were analyzed using a t-test $(\mathrm{P}=5.5 \mathrm{e}-29) ;{ }^{* * *} \mathrm{P}<0.001$. (D) Expression of LINC02454 in PTC tissues and matched normal tissues in GEO data. Data were analyzed using a paired t-test $(\mathrm{P}=2.64 \mathrm{e}-05) ;{ }^{* * *} \mathrm{P}<0.001$. $\mathrm{PTC}$, papillary thyroid cancer.

54 paired PTC and adjacent normal thyroid tissues. These results revealed that all 6 lncRNAs were upregulated in the PTC tissues compared with the normal adjacent tissues, which is consistent with the expression levels in the lncRNA microarray. The levels of ENST00000417422.1, ENST00000457989.1, ENST00000539116.1, and ENST00000539653.1 were markedly higher in the PTC tissues compared with the normal adjacent tissues $(\log \mathrm{FC}>2 ; \mathrm{P}<0.05)$ (Fig. S2), while 3 of these IncRNAs (ENST00000539653, ENST00000417422.1 and ENST00000457989.1) have also been reported to be involved in PTC in a previous study (35). However, the biological function of ENST00000539116.1 (a transcript of LINC02454) has not been previously reported, at least to the best of our knowledge; therefore, LINC02454 was selected for further investigation.

Expression of LINC02454 in PTC. To explore the expression level of LINC02454 in PTC cell lines and tissues, RT-qPCR was performed in 104 paired tumor and normal control samples. A total of 4 representative pathological images of PTC and normal control samples are shown in Fig. S3. The LINC02454 expression level was significantly increased in the tumor tissues compared with the normal control samples $(\mathrm{P}=0.0011$; Fig. 2A). In addition, the LINC02454 levels were also examined in the TPC1 and IHH4 cell lines. A strong expression of LINC02454 was detectable in both cell lines, while the expression level was higher in the TPC1 cell line (the lower the $\Delta \mathrm{Ct}$ value, the higher the expression); therefore, it was selected for further functional analysis ( $\mathrm{P}=0.0136$; Fig. 2B). As shown in Fig. 2C, LINC02454 was significantly upregulated in PTC $(n=502)$ with a $\operatorname{logFC}$ of 4.16 and a $\mathrm{P}=5.5 \times 10^{-29}$ compared with the normal samples $(n=58)$ in the TCGA database. The LINC02254 expression level was also verified in PTC with the GEO dataset. It was found that ENST00000539116.1 was significantly upregulated in the cancer samples $(n=5)$ compared with the matched non-cancerous samples $\left(\mathrm{P}=2.64 \times 10^{-5}\right)$ (Fig. 2D), which is consistent with the results obtained from TCGA database and the microarray data.

LINC02454 expression and clinicopathological characteristics. The clinical significance of LINC02454 was investigated by analyzing the association of LINC02454 expression in 104 patients with clinicopathological characteristics, including 
Table II. Association between the LINC02454 expression level and patient clinicopathological characteristics.

\begin{tabular}{|c|c|c|c|}
\hline \multirow[b]{2}{*}{ Variables } & \multicolumn{2}{|c|}{ LINC02454 subgroup $^{a}$} & \multirow[b]{2}{*}{ P-value } \\
\hline & Low $[\mathrm{n}(\%)]$ & $\operatorname{High}[\mathrm{n}(\%)]$ & \\
\hline All cases & 52 & 52 & \\
\hline Age (years) & & & 0.163 \\
\hline$<55$ & $43(53.8)$ & $37(46.3)$ & \\
\hline$\geq 55$ & $9(37.5)$ & $15(62.5)$ & \\
\hline Sex & & & 0.365 \\
\hline Male & $15(57.7)$ & $11(42.3)$ & \\
\hline Female & $37(47.4)$ & $41(52.6)$ & \\
\hline Maximum size tumor $(\mathrm{cm})$ & & & $0.031^{\mathrm{c}}$ \\
\hline$<2$ & $30(61.2)$ & $19(38.8)$ & \\
\hline$\geq 2$ & $22(40.0)$ & $33(60.0)$ & \\
\hline Multifocality & & & 0.168 \\
\hline Unifocal & $32(56.1)$ & $25(43.9)$ & \\
\hline Multifocal & $20(42.6)$ & $27(57.4)$ & \\
\hline ETE & & & 0.116 \\
\hline No & $28(58.3)$ & $20(41.7)$ & \\
\hline Yes & $24(42.9)$ & $32(57.1)$ & \\
\hline T stage & & & $0.006^{\mathrm{d}}$ \\
\hline $\mathrm{T} 1-\mathrm{T} 2$ & $32(64.0)$ & $18(36.0)$ & \\
\hline T3-T4 & $20(37.0)$ & $34(63.0)$ & \\
\hline LNM & & & $0.020^{\mathrm{c}}$ \\
\hline N0 & $22(66.7)$ & $11(33.3)$ & \\
\hline $\mathrm{N} 1$ & $30(42.3)$ & $41(57.7)$ & \\
\hline TNM stage & & & $0.046^{\mathrm{c}}$ \\
\hline I-II & $50(53.2)$ & $44(46.8)$ & \\
\hline III-IV & $2(20.0)$ & $8(80.0)$ & \\
\hline
\end{tabular}

The percentages shown in the table were calculated out of the total numbers of patients with a low and high expression for each sub-variable. ${ }^{a}$ Median expression level was used as a cut-off to divide the 104 patients into the LINC02454 low group ( $\mathrm{n}=52$ ) and LINC02454 high group $(\mathrm{n}=52)$; ${ }^{\mathrm{b}} \mathrm{Chi}$-square test; ${ }^{\mathrm{c}} \mathrm{P}<0.05$; ${ }^{\mathrm{d}} \mathrm{P}<0.01$. ETE, extrathyroidal extension; LNM, lymph node metastasis; TNM, tumor-node metastasis.

sex, age, tumor size, multimodality, lymph node metastasis (LNM), extra-thyroidal extension (ETE), T stage and TNM stage. Subsequently, 104 patients with PTC were categorized into 2 groups (LINC02454 high and low expression groups) according to the median relative LINC02454 expression value $(\Delta \mathrm{Ct}=10.283)$. The LINC02454 high expression group was significantly associated with a larger tumor volume, higher $\mathrm{T}$ stage, a more advanced TNM stage and a higher number of lymph node metastasis $(\mathrm{P}<0.05$; Table II), but not with sex, age, multimodality, or extrathyroidal extension (ETE) (P>0.05; Table II). From the analysis of the TCGA data, the expression level of LINC02454 was significantly associated with clinical traits, such as lymph node count, pathological stage and TNM stage (Fig. 3). The median value of lymph node count was used to divide the samples into the high $(n=190)$ and low $(n=198)$ lymph nodes groups. LINC02454 was significantly upregulated in the high lymph node group $(\mathrm{P}=0.01836$; Fig. $3 \mathrm{~A})$. In addition, LINC02454 was significantly upregulated in N1 stage $(n=223)$ $\left(\mathrm{P}=3.843 \times 10^{-12}\right)$ compared with N0 stage $(\mathrm{n}=229)$ (Fig. 3B). The groups T1 and T2 were combined into one group $(n=307)$, while T3 and T4 was combined into another group $(n=193)$. LINC02454 was significantly upregulated in stages T3 and 4 $(\mathrm{P}=0.0003377)$ (Fig. 3C). Stages I and II were also combined into one group $(\mathrm{n}=333)$, while stages III and IV where also combined into another group $(n=167)$. LINC02454 was significantly upregulated in stages III and IV $(\mathrm{P}=0.00083355)$ (Fig. 3D). The findings from TCGA database were consistent with the results from RT-qPCR.

High LINC02454 expression is associated with BRAF mutation and a poorer disease-free survival in the TCGA PTC cohort. It was also found that LINC02454 was significantly upregulated in patients with BRAF mutations $(n=239)$ compared with patients without BRAF mutations $(n=263)$ $(\mathrm{P}<0.001$; Fig. 4A). As shown in Fig. S4, based on the ROC, an optimal threshold of 1.742 for LINC02454 expression level was obtained by optimizing the Youden's index. A total of 484 PTC patients were classified into 2 groups (408 high and 76 low levels of LINC02454 expression groups) based on the Youden's index. The sensitivity, specificity, and positive 
A

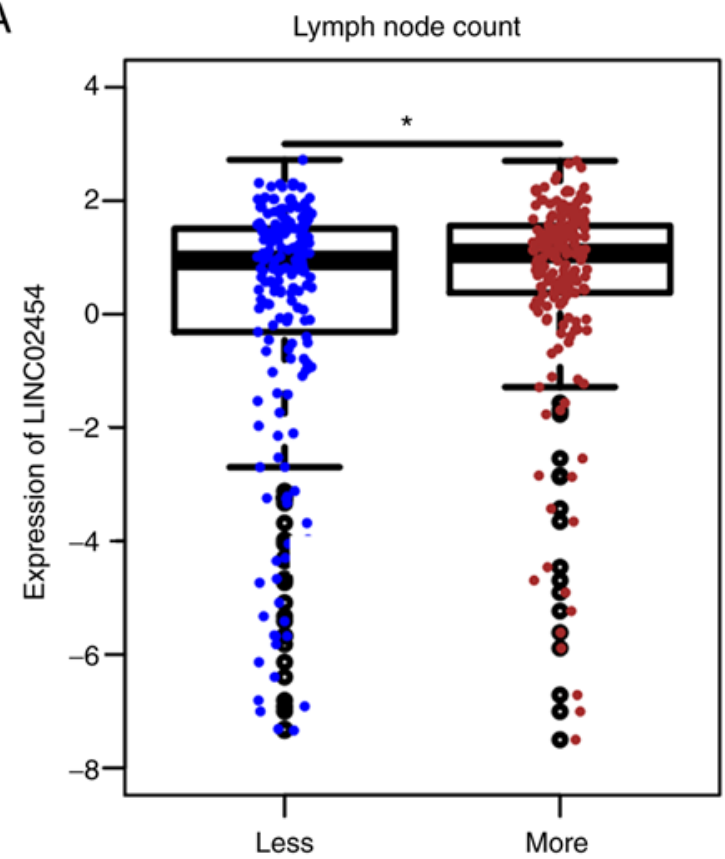

C

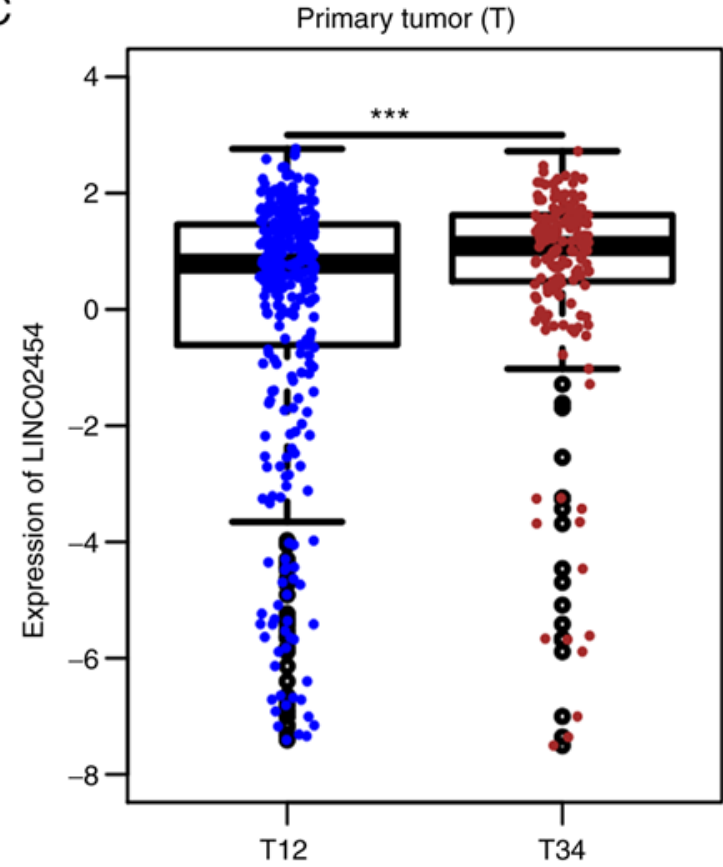

B

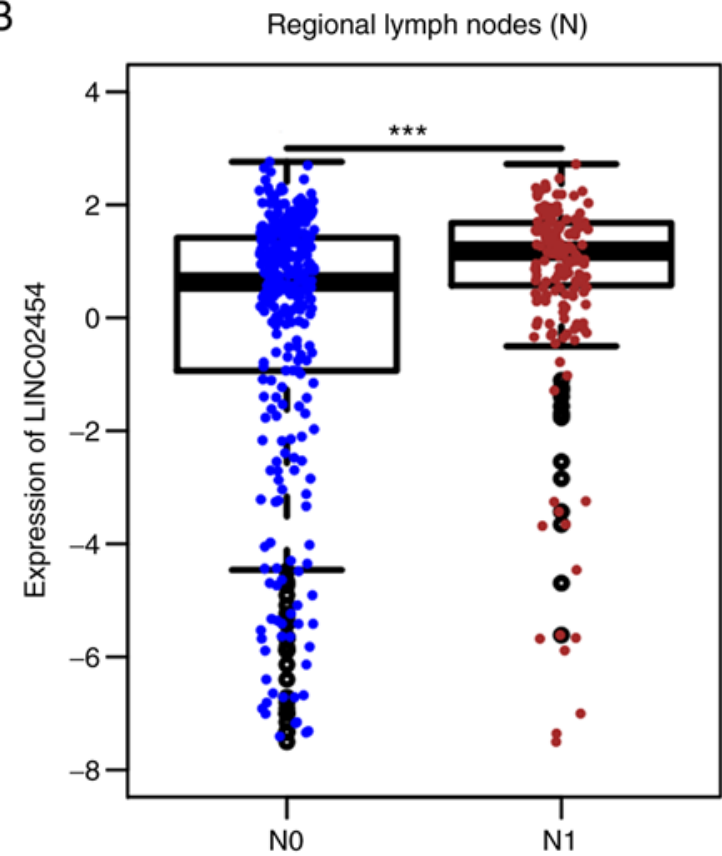

D

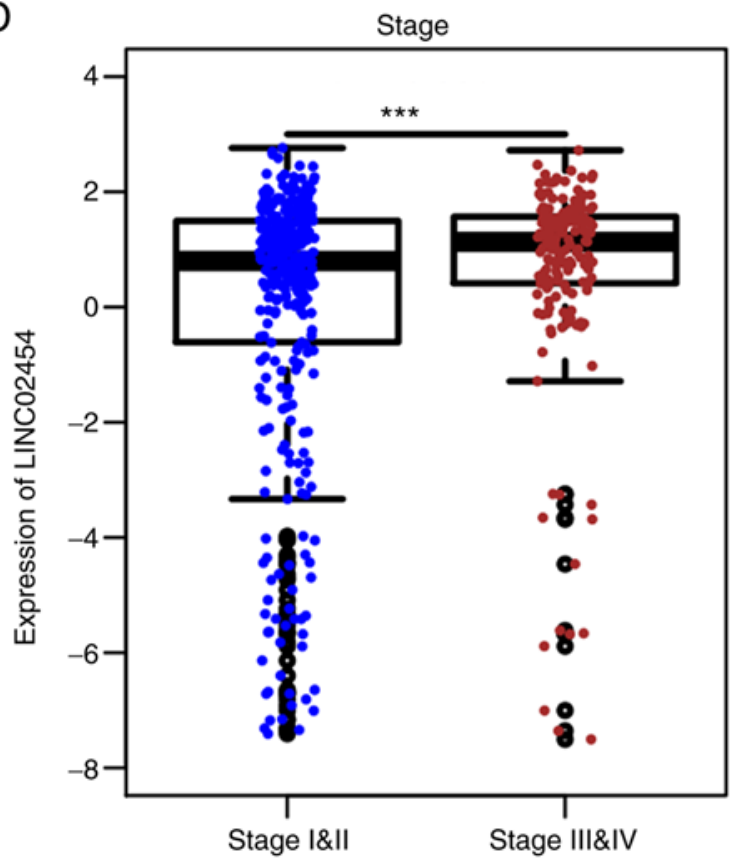

Figure 3. Association of LINC02454 expression with clinical traits of patients with PTC in the TCGA data. LINC02454 upregulation was associated with (A) a higher lymph node count $(\mathrm{P}=0.01836),(\mathrm{B})$ an advanced $\mathrm{N}$ stage $(\mathrm{P}=3.843 \mathrm{e}-12),(\mathrm{C})$ an advanced $\mathrm{T}$ stage $(\mathrm{P}=0.0003377)$ and $(\mathrm{D})$ an advanced pathological stage $(\mathrm{P}=0.00083355)$. Data were analyzed using a t-test; ${ }^{*} \mathrm{P}<0.05$ and ${ }^{* * * *} \mathrm{P}<0.001$. $\mathrm{PTC}$, papillary thyroid cancer.

diagnostic likelihood ratio of 1.742 for disease-free survival diagnosis were $34.8 \%, 86.1 \%$ and 2.498 , respectively, with an area under the curve at 0.596 (95\% CI, 0.505-0.687). In addition, high expression levels of LINC02454 were significantly associated with a poorer disease-free survival $(\mathrm{P}=0.000358)$ (Fig. 4B). In addition, as shown in Fig. S5, the poor disease-free survival time of patients with PTC was associated with an advanced $\mathrm{N}$ stage $(\mathrm{P}=0.03860)$, advanced $\mathrm{T}$ stage $(\mathrm{P}=0.00205)$ and an advanced pathological stage $(\mathrm{P}=0.000827)$. No significant associations were observed between the disease-free survival time of patients with PTC and lymph node count $(\mathrm{P}=0.53400)$.
Identification of biological functions of LINC02454-related genes. To reveal the biological properties of LINC02454, 502 PTC samples from TCGA were divided into the high and low expression groups based on the median LINC02454 expression levels (3.151). Subsequently, 531 differentially expressed genes were identified, which included 194 down- and 337 upregulated genes in the LINC02454 high expression group compared with the LINC02454 low expression group (Fig. 5). The PPI network consisted of 50 nodes and 124 edges, which included 22 down- and 98 upregulated genes (Fig. 6). Following which, functional enrichment analyses was performed using the LINC02454-related genes, which revealed that the 
A

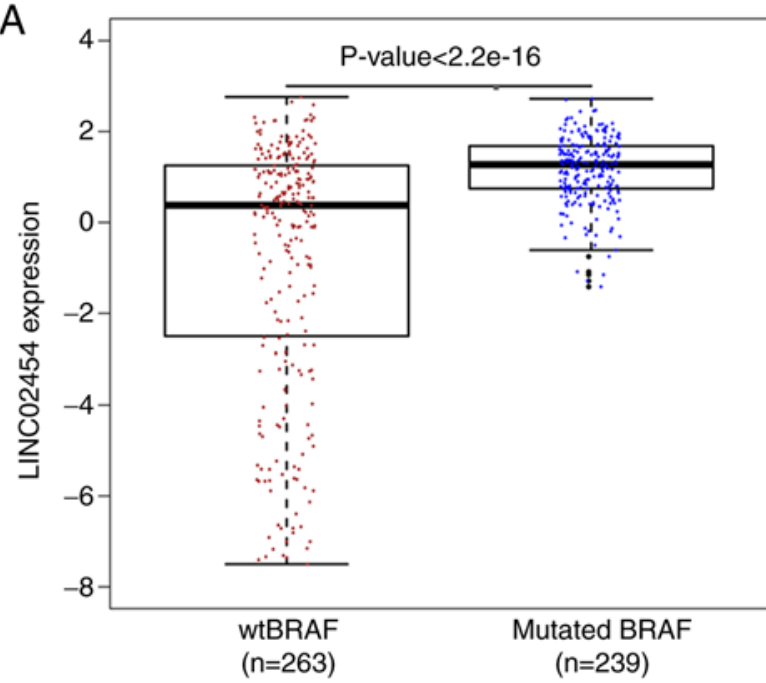

B

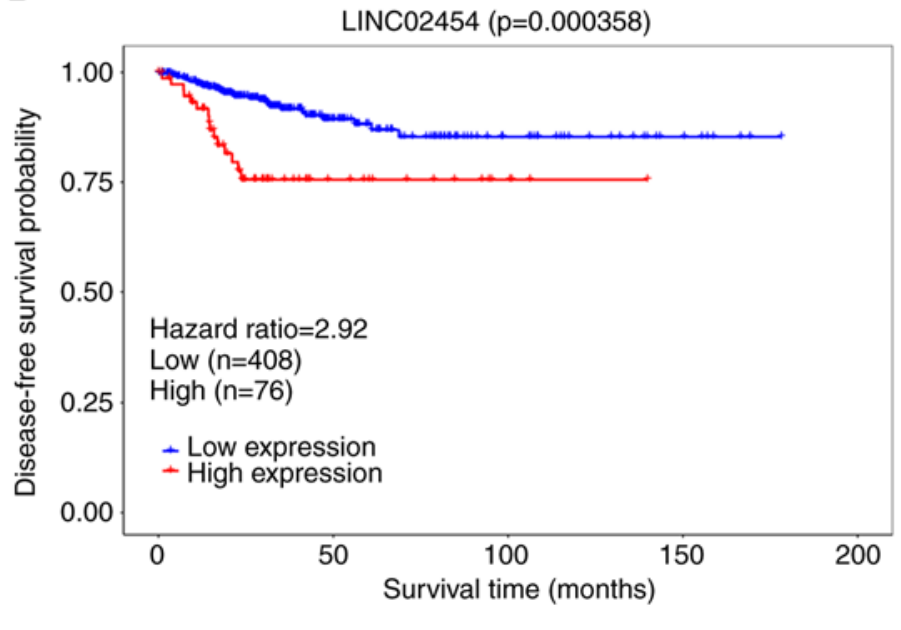

Figure 4. Association of LINC02454 expression with BRAF mutation and disease-free survival in the TCGA PTC cohort. (A) Expression of LINC02454 in patients with PTC with BRAF mutation and wtBRAF. Data were analyzed using a t-test $(\mathrm{P}=2.2 \mathrm{e}-16)$. (B) Disease-free survival time in the high and low LINC02454 expression groups; $\mathrm{n}=484$, (log-rank test, $\mathrm{P}=0.000358)$. PTC, papillary thyroid cancer.

upregulated genes were primarily enriched in the KEGG pathways, such as 'pathways in cancer', 'proteoglycans in cancer' and 'ECM-receptor interaction' and in the GO BP terms, such as 'positive regulation of gene expression', 'angiogenesis', 'positive regulation of cell proliferation', 'cell adhesion' and 'positive regulation of cell division', while the downregulated genes were primarily enriched in the metabolic pathways (Fig. 7). The following enriched terms were subsequently investigated: Cell proliferation, cell division and pathways in cancer.

Interference efficiency of LINC02454 siRNAs in TPC1 cells. To investigate the potential biological functions of LINC02454, it was knocked down using 3 siRNAs and a negative control in the TPC1 cell line (Table I). The expression level of LINC02454 in the TPC1 cells was quantified by RT-qPCR at $48 \mathrm{~h}$ following siRNA transfection. When compared to the cells transfected with siRNA-NC, the expression levels of LINC02454 in the si-LINC02454-1- $(\mathrm{P}<0.01)$, si-LINC02454-2- $(\mathrm{P}<0.01)$ and si-LINC02454-3-transfected $(\mathrm{P}<0.01)$ TPC1 cells were decreased (Fig. 8). The interference efficiency of si-LINC02454 was successfully achieved (65-85\%), with the highest efficiency found with si-LINC02454-2. Therefore, it was selected for further use in knockdown experiments.

Effect of LINC02454 on in vitro TPC1 cell production. To investigate the in vitro effect of LINC02454 on cell proliferation, the TPC1 cells were subjected to colony formation and CCK-8 assays following transfection with si-LINC02454-2 and si-NC. The proliferation of the TPC1 cells was significantly decreased following transfection with si-LINC02454-2 compared with that of the si-NC-transfected cells $(\mathrm{P}<0.05$; Fig. 9A). In addition, the silencing of LINC02454 significantly reduced colony numbers compared with the $\mathrm{NC}$ group ( $\mathrm{P}<0.01$; Fig. 9B). Based on these findings, it was hypothesized that LINC02454 may play a critical role in regulating PTC cell growth and proliferation.

Effect of LINC02454 on the cell cycle of TPC1 cell lines in vitro. To investigate whether LINC02454 affects the

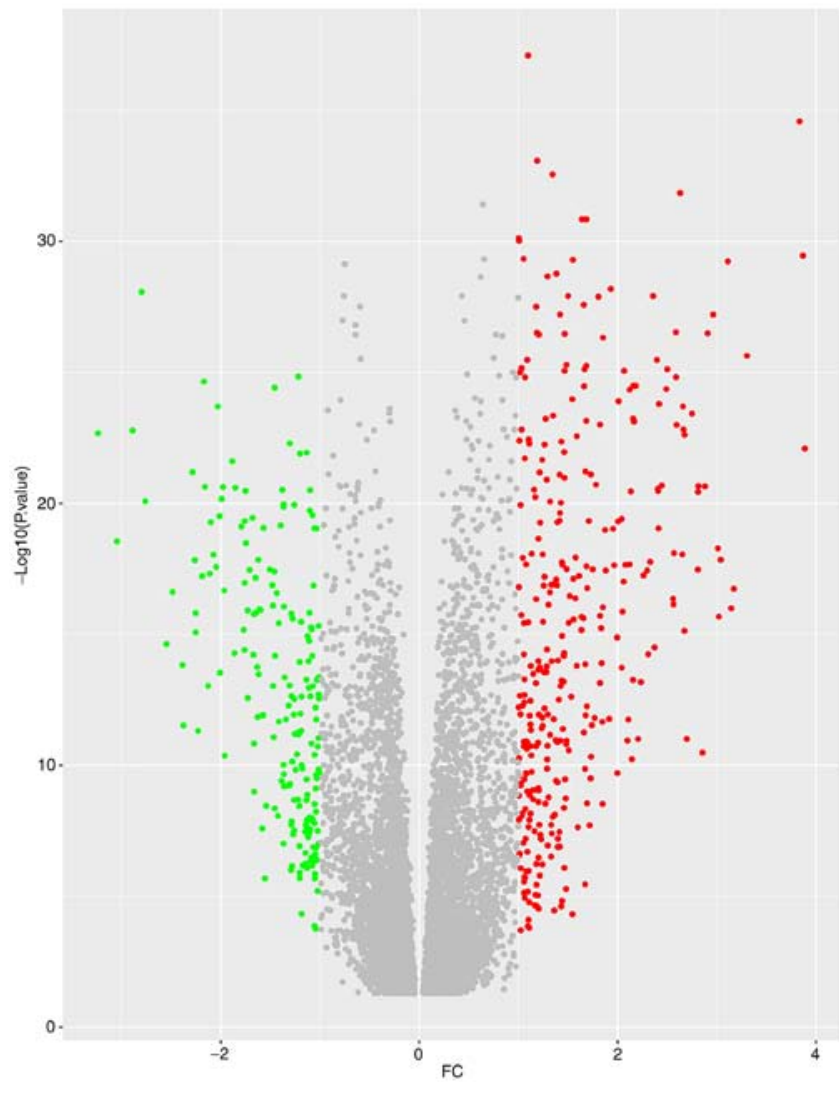

Figure 5. Volcano plot of the differentially expressed genes in the high and low LINC02454 expression groups. Green dots indicate downregulated genes, while red dots indicate upregulated genes.

distribution of TPC1 cells in the cell cycle in vitro, the cell cycle profile of TPC1 cells was determined at $48 \mathrm{~h}$ following transfection with si-LINC02454-2 and si-NC by flow cytometric analysis. The results revealed that there was a decrease in cell numbers in the $S$ phase and an increase in cells in $G_{0} / G_{1}$ in cells transfected with si-LINC02454-2 compared with the cells transfected with si-NC $(\mathrm{P}<0.05$ and $\mathrm{P}<0.01$; Fig. 9C). 


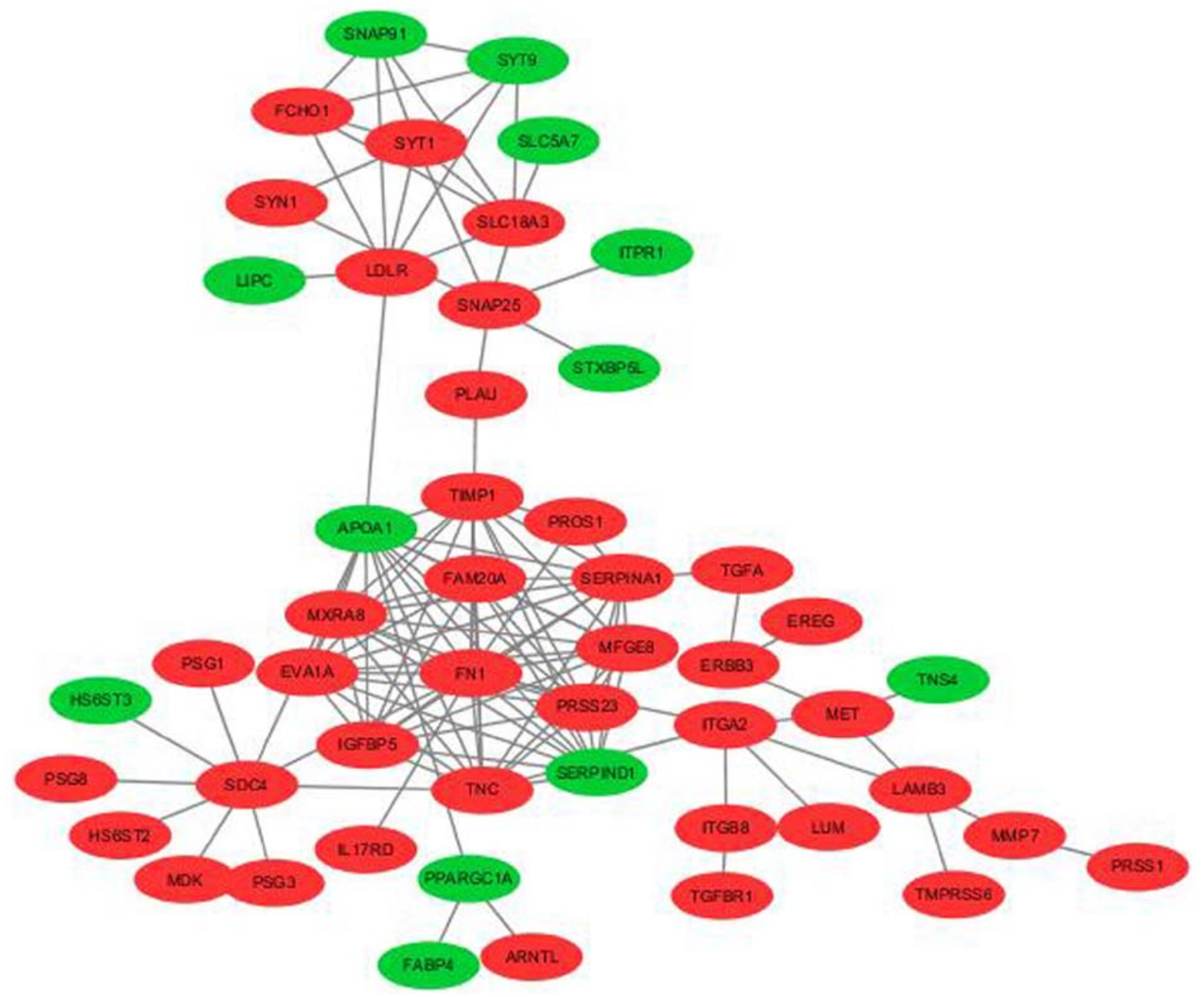

Figure 6. PPI network of differentially expressed genes in high and low LINC02454 expression groups. Red nodes represent upregulated genes, while green nodes represent downregulated genes.

Effect of LINC02454 on the apoptosis of TPC1 cell lines in vitro. The effect of LINC02454 on TPC1 cell apoptosis was investigated using an apoptosis assay and flow cytometry. Apoptotic cells were considered to consist of early and late apoptotic cells. There was a significant increase in the percentages of apoptotic cells in the si-LINC02454-transfected cells compared with the si-NC-transfected TPC1 cells ( $\mathrm{P}<0.05$; Fig. 9D), which suggests that LINC02454 is involved in regulating TPC1 cell cycle progression and apoptosis.

\section{Discussion}

Over the past few decades, several IncRNAs have been demonstrated to be significant regulators of thyroid tumorigenesis. Moreover, abnormal expression levels of cancer-related lncRNAs have led to the conclusion that such IncRNAs can be either tumor promoters or suppressors, that can exert various effects on their target genes. In the present study, by lncRNA expression microarray profiling, it was shown that LINC02454 was significantly upregulated in PTC tissues compared with normal control tissues. LINC02454, a 589 bp RNA located on $12 \mathrm{q} 14.3$, is a novel gene LINCRNA, that has not been fully researched. In the present study, the biological roles of LINC02454 in PTC were explored.
Firstly, in the present study, PTC tissues had a significantly higher LINC02454 expression level compared with that in control tissue samples. An extensive overlapping compared to normal tissue was also found; however, there was a marked increase in LINC02454 expression in the TCGA and GEO cohort data. There are 3 reasons to account for this. One is the different methods used to calculate the expression of LINC02454 in the 3 cohort data. In the present study, the $\Delta \mathrm{Ct}$ method (2) was used to calculate the relative expression levels of LINC02454. In the TCGA database, the expression of LINC02454 was obtained by normalizing the counts of the gene using the zoom method. In the GEO 66783 database, the expression of LINC02454 was directly obtained from the GSE66783_series_matrix.txt (GEO database) file. Another reason is different racial types. The present study was based on an Asian population. In the TCGA cohort, the study subjects were Caucasians, African Americans and Asians. The third is the different experimental methods. RT-qPCR was used to detect the expression of LINC02454 in the present study, RNA-seq sequencing in the TCGA cohort, and Chip technology in the GSE66783 cohort. Therefore, LINC02454 may be a beneficial diagnostic marker in PTC. The expression levels of LINC02454 were associated with clinical characteristics and a poor disease-free survival of patients 


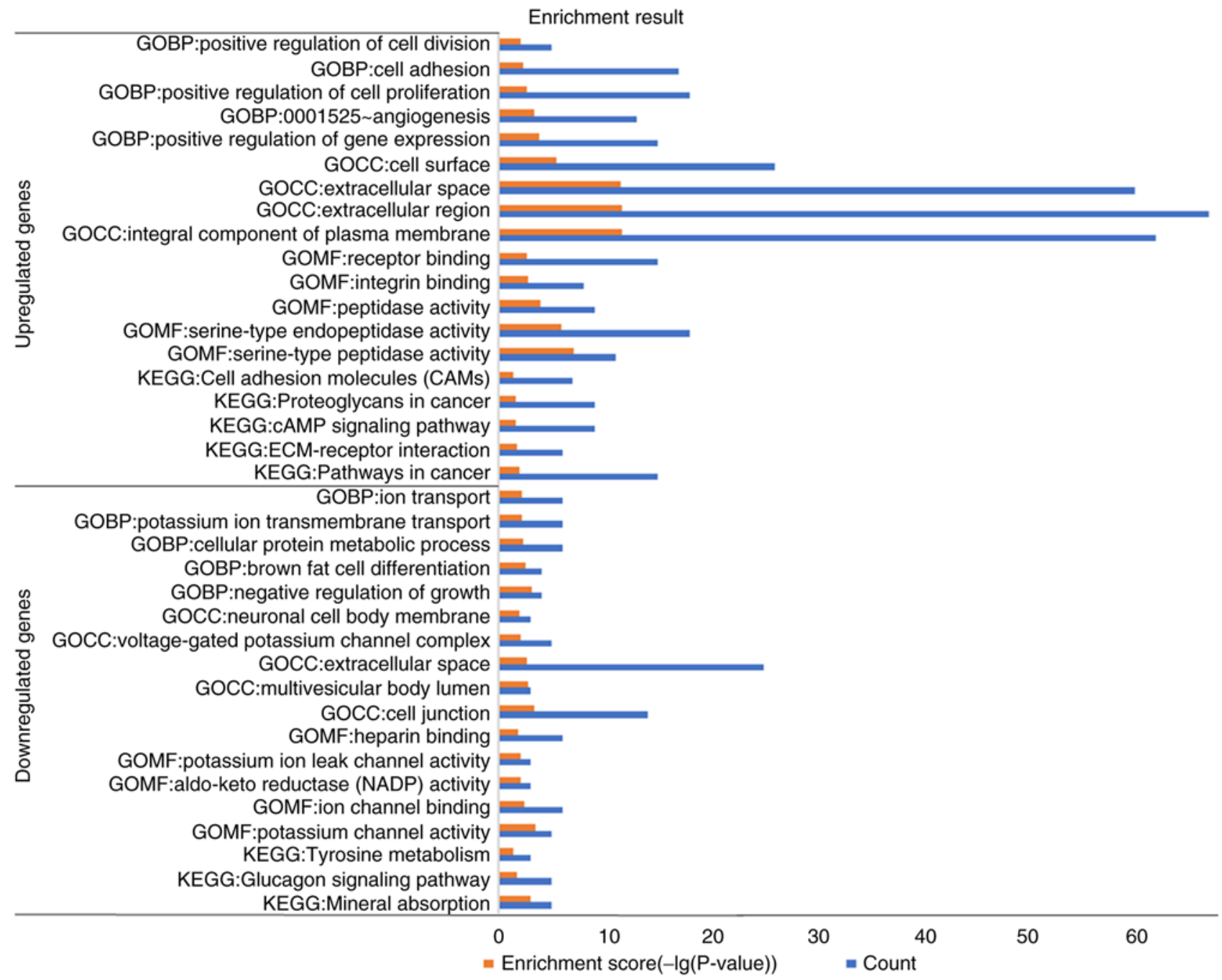

Figure 7. Enriched molecular function (MF), biological process (BP) and cellular component (CC) terms and the Kyoto Encyclopedia of Genes and Genomes (KEGG) pathways for differentially expressed genes in high and low LINC02454 expression groups.

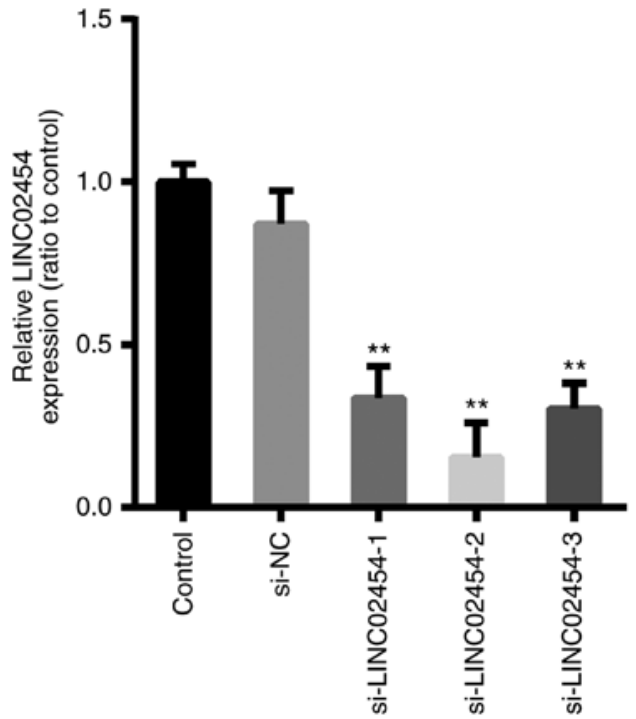

Figure 8. RNA interference (siRNA) decreased the expression of LINC02454 in TPC1 cells. The expression levels of LINC02454 in TPC1 cells transfected with si-LINC02454-1, si-LINC02454-2, and si-LINC02454-3 were significantly decreased compared with those in the siRNA-NC groups; ${ }^{* *} \mathrm{P}<0.01$, compared to the control. with PTC. It was hypothesized that LINC02454 may be an oncogene for PTC. Thus, to investigate the role of LINC02454 in PTC, functional assays were performed. First, functional enrichment analysis of LINC02454-related genes revealed that these genes were enriched in pathways, such as 'pathways in cancer', 'cell proliferation' and 'cell division', indicating that LINC02454 may play a critical role in cell proliferation and the cell cycle. Hence, cell proliferation and apoptosis assays were performed to investigate whether LINC02454 affects the proliferation processes of PTC cell lines. The results indicated that the knockdown of LINC02454 resulted in an increase in apoptosis, while decreasing cellular proliferation rates in vitro. These findings suggest that LINC02454 may act as an oncogene in the development of PTC.

In addition, the underlying mechanisms of the association between LINC02454 expression levels and aggressive clinical outcomes and a poor disease-free survival of patients with PTC was investigated. PTC is the second most common type of cancer following melanoma with the BRAFV600E mutation. Previous studies have demonstrated that BRAF (V600E) mutations are associated with lymph node and distant tumor metastasis, and the survival of patients with PTC $(36,37)$. In the present study, it 
A

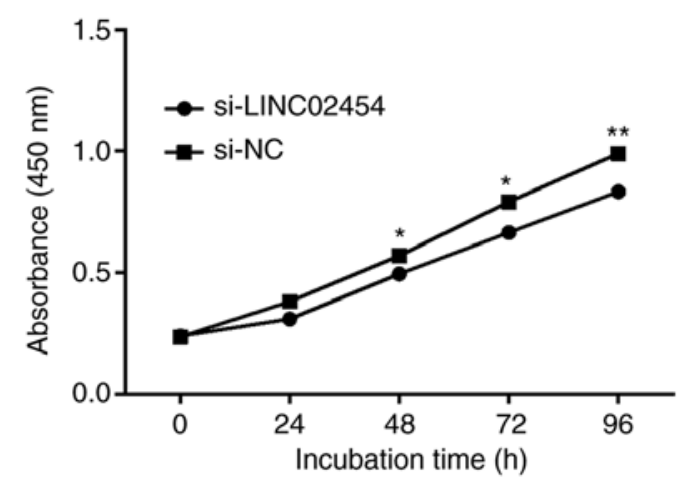

B

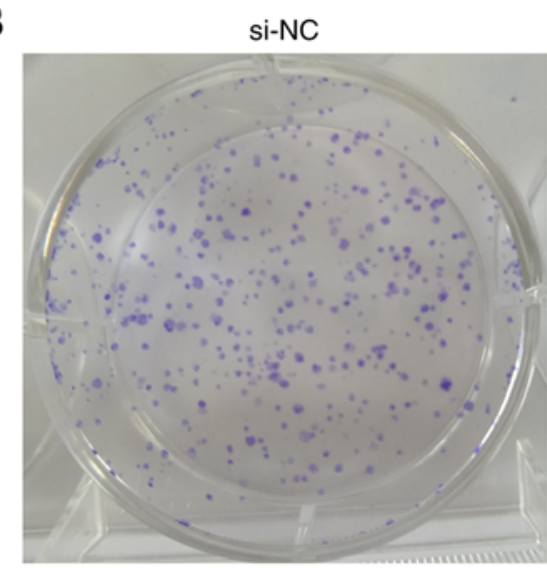

C

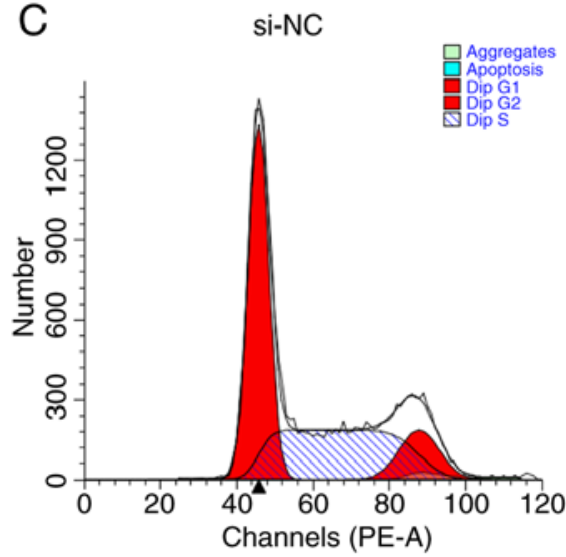

D

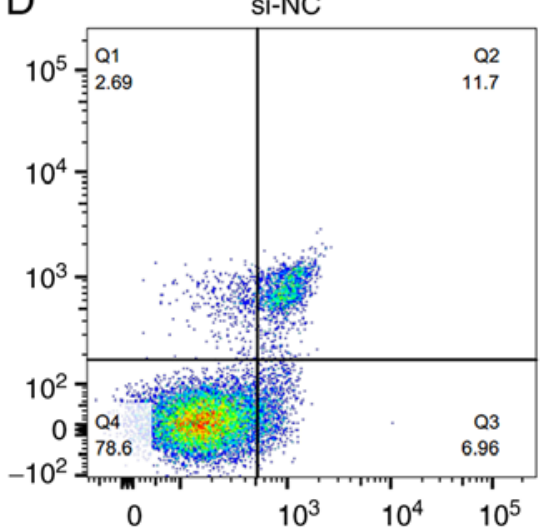

si-LINC02454
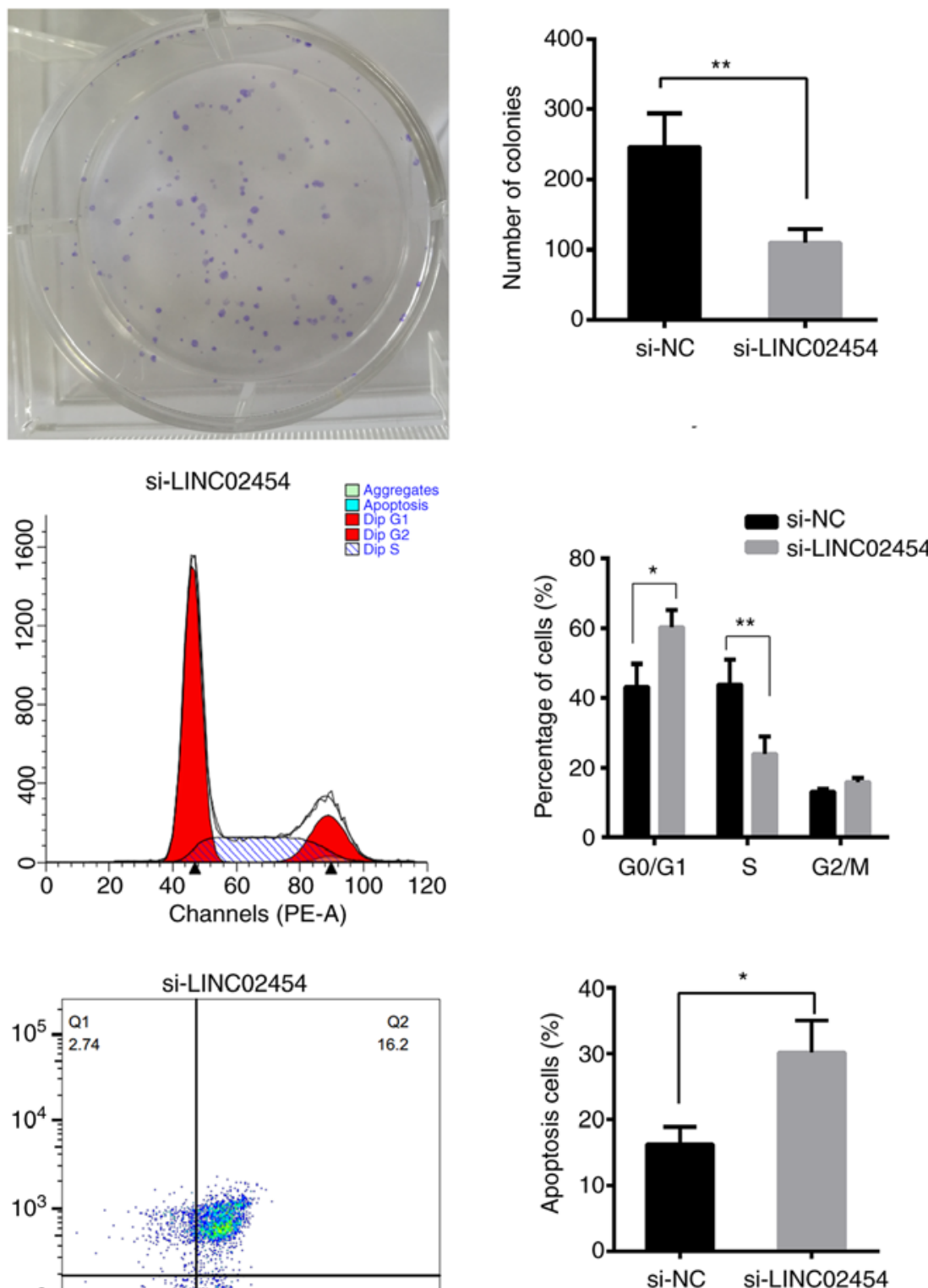

Figure 9. Downregulation of LINC02454 in TPC1 cells inhibits cell proliferation in vitro. (A) effects of LINC02454 on the proliferation of the TPC1 cell line. The proliferation capability of TPC1 cells was significantly decreased following transfection with si-LINC02454 compared with the si-NC group ( $<0.05)$. (B) Colony formation assay assessed the ability of LINC02454 to form colonies $(\mathrm{P}<0.01)$. Data are expressed as the median (interquartile range) from 3 independent experiments performed in triplicate. (C) Effects of LINC02454 on the cell cycle of TPC1 cell lines. The percentage cells in G0/G1 phase was increased $(\mathrm{P}<0.05)$, while the percentage of cells in the $\mathrm{S}$ phase $(\mathrm{P}<0.01)$ was markedly decreased following transfection with si-LINC02454 compared to the si-NC groups. (D) Function of LINC02454 in TPC1 cell apoptosis. TPC1 cells underwent apoptosis at a higher rate at $48 \mathrm{~h}$ following transfection compared with those transfected with si-NC $(\mathrm{P}<0.05)$. ${ }^{*} \mathrm{P}<0.05$ and ${ }^{* *} \mathrm{P}<0.01$. Values represent the means $\pm \mathrm{SD}$ from 3 independent experiments performed in triplicate. 
was found that patients with the BRAF (V600E) mutation had significantly increased expression levels of LINC02454 in the TCGA cohort, suggesting that LINC02454 may be one of the BRAF (V600E)-related lncRNAs, and may be possibly involved in BRAF (V600E)-induced PTC development. The BRAF (V600E) mutation is the most frequently mutated gene that activates the MAPK signaling pathway $(38,39)$. Thyroid tumorigenesis is highly dependent on the MAPK pathway, which is a critical transduction pathway. The aberrant activation of this cascade affects cell proliferation, differentiation, migration, senescence and apoptosis $(40,41)$. In addition, a previous study found that the AKT/mTOR pathway was overactivated in PTC, and harbored the BRAF (V600E) mutation (42). In addition, novel BRAF mutation-related genes have been identified in PTC (e.g., MMD, ITPR3, AACS, LAD1, PVRL3, ALDH3B1 and RASA1), which will be used as a basis for future research (43).

In conclusion, to the best of our knowledge, this is the first study to demonstrate that LINC02454 is highly expressed in thyroid cancer tissues and functions as an oncogene in PTC. Furthermore, it was found to be associated with an aggressive clinical outcome and a poor disease-free survival of patients with PTC. The findings of the present study provides insight into the applications of LINC02454 for the diagnosis, prognosis or treatment of PTC. However, the present study only performed in vitro studies; therefore, further investigations are required to discover the underlying mechanisms involved in the suppression of growth and increase in apoptosis following knockdown of LINC02454 in TPC1 cells.

\section{Acknowledgements}

The authors gratefully acknowledge the assistance of Dr Chao Liu of Nanjing University of Chinese Medicine, who provided the valuable papillary thyroid cancer cell lines used in the present study. The authors also owe a special debt of gratitude to Ross Ihaka and Robert Gentleman of the Statistics Department of the University of Auckland, who created the R programming language.

\section{Funding}

The present study was supported in part by the Key Research and Development Project of Jiangsu Province (BE2015723), Six Talent Peak Funding Plan (WSN-061), the Technology Development Fund of Nanjing Medical University (NMUB2018144) and the Municipal Science and Technology Plan Project of Huai'an.

\section{Availability of data and materials}

The datasets used during the present study are available from the corresponding author upon reasonable request.

\section{Authors' contributions}

YL, XY and JT designed the study. JT, LL, TC, BS, DD and $\mathrm{ZZ}$ collected the data and performed the analysis. YL, XY and DD supervised the study, and contributed to the drafting of the manuscript. JT drafted the manuscript. All authors read and approved the final manuscript.

\section{Ethics approval and consent to participate}

Study protocols were reviewed and pre-approved by the Ethics Committee of the Second Affiliated Hospital of Nanjing Medical University (no. 2014 KY No. 054). All patients provided written informed consent prior to participation in this study.

\section{Patient consent for publication}

Not applicable.

\section{Competing interests}

The authors declare that they have no competing interests.

\section{References}

1. Bray F, Ferlay J, Soerjomataram I, Siegel RL, Torre LA and Jemal A: Global cancer statistics 2018: GLOBOCAN estimates of incidence and mortality worldwide for 36 cancers in 185 countries. CA Cancer J Clin 68: 394-424, 2018.

2. Pusztaszeri M and Auger M: Update on the cytologic features of papillary thyroid carcinoma variants. Diagn Cytopathol 45: 714-730, 2017.

3. Chen W, Zheng R, Baade PD, Zhang S, Zeng H, Bray F, Jemal A, $\mathrm{Yu}$ XQ and He J: Cancer statistics in China, 2015. CA Cancer J Clin 66: 115-132, 2016.

4. Liyanarachchi S, Li W, Yan P, Bundschuh R, Brock P, Senter L, Ringel MD, de la Chapelle A and $\mathrm{He} \mathrm{H}$ : Genome-wide expression screening discloses long noncoding RNAs involved in thyroid carcinogenesis. J Clin Endocrinol Metab 101: 4005-4013, 2016.

5. Chen W, Zheng R, Zeng H, Zhang S and He J: Annual report on status of cancer in China, 2011. Chin J Cancer Res 27: 2-12, 2015.

6. Roman BR, Morris LG and Davies L: The thyroid cancer epidemic, 2017 perspective. Curr Opin Endocrinol Diabetes Obes 24: 332-336, 2017.

7. Karadaghy OA, Kallogjeri D and Piccirillo JF: Development of a new clinical severity staging system for patients with nonmetastatic papillary thyroid carcinoma. JAMA Otolaryngol Head Neck Surg 143: 1173-1180, 2017.

8. Grogan RH, Kaplan SP, Cao H, Weiss RE, Degroot LJ, Simon CA, Embia OM, Angelos P, Kaplan EL and Schechter RB: A study of recurrence and death from papillary thyroid cancer with 27 years of median follow-up. Surgery 154: 1436-1447, 2013.

9. Giordano TJ, Kuick R, Thomas DG, Misek DE, Vinco M, Sanders D, Zhu Z, Ciampi R, Roh M, Shedden K, et al: Molecular classification of papillary thyroid carcinoma: Distinct BRAF, RAS, and RET/PTC mutation-specific gene expression profiles discovered by DNA microarray analysis. Oncogene 24: 6646-6656, 2005.

10. Adeniran AJ, Zhu Z, Gandhi M, Steward DL, Fidler JP, Giordano TJ, Biddinger PW and Nikiforov YE: Correlation between genetic alterations and microscopic features, clinical manifestations, and prognostic characteristics of thyroid papillary carcinomas. Am J Surg Pathol 30: 216-222, 2006.

11. Greco A, Miranda C and Pierotti MA: Rearrangements of NTRK1 gene in papillary thyroid carcinoma. Mol Cell Endocrinol 321: 44-49, 2010.

12. Ellis RJ, Wang Y, Stevenson HS, Boufraqech M, Patel D, Nilubol N, Davis S, Edelman DC, Merino MJ, He M, et al: Genome-wide methylation patterns in papillary thyroid cancer are distinct based on histological subtype and tumor genotype. J Clin Endocrinol Metab 99: E329-E337, 2014.

13. Murugan AK, Munirajan AK and Alzahrani AS: Long noncoding RNAs: Emerging players in thyroid cancer pathogenesis. Endocr Relat Cancer 25: R59-R82, 2018.

14. Sui F, Ji M and Hou P: Long non-coding RNAs in thyroid cancer: Biological functions and clinical significance. Mol Cell Endocrinol 469: 11-22, 2018.

15. Wei MM and Zhou GB: Long Non-coding RNAs and their roles in Non-small-cell lung cancer. Genomics Proteomics Bioinformatics 14: 280-288, 2016. 
16. Bartonicek N, Maag JL and Dinger ME: Long noncoding RNAs in cancer: Mechanisms of action and technological advancements. Mol Cancer 15: 43, 2016.

17. Li JH, Zhang SQ, Qiu XG, Zhang SJ, Zheng SH and Zhang DH: Long non-coding RNA NEAT1 promotes malignant progression of thyroid carcinoma by regulating miRNA-214. Int J Oncol 50: 708-716, 2017.

18. Kim D, Lee WK, Jeong S, Seol MY, Kim H, Kim KS, Lee EJ, Lee $\mathrm{J}$ and Jo YS: Upregulation of long noncoding RNA LOC100507661 promotes tumor aggressiveness in thyroid cancer. Mol Cell Endocrinol 431: 36-45, 2016.

19. Yang T, Zhai H, Yan R, Zhou Z, Gao L and Wang L: IncRNA CCAT1 promotes cell proliferation, migration, and invasion by down-regulation of miR-143 in FTC-133 thyroid carcinoma cell line. Braz J Med Biol Res 51: e7046, 2018.

20. Dai W, Tian Y, Jiang B and Chen W: Down-regulation of long non-coding RNA AFAP1-AS1 inhibits tumor growth, promotes apoptosis and decreases metastasis in thyroid cancer. Biomed Pharmacother 99: 191-197, 2018.

21. Zheng H, Wang M, Jiang L, Chu H, Hu J, Ning J, Li B, Wang D and $\mathrm{Xu}$ J: BRAF-activated long noncoding RNA modulates papillary thyroid carcinoma cell proliferation through regulating thyroid stimulating hormone receptor. Cancer Res Treat 48: 698-707, 2016.

22. Wang C, Yan G, Zhang Y, Jia X and Bu P: Long non-coding RNA MEG3 suppresses migration and invasion of thyroid carcinoma by targeting of Rac1. Neoplasma 62: 541-549, 2015.

23. Ma B, Liao T, Wen D, Dong C, Zhou L, Yang S, Wang Y and Ji Q: Corrigendum: Long intergenic non-coding RNA 271 is predictive of a poorer prognosis of papillary thyroid cancer. Sci Rep 7: 42321, 2017.

24. Lan X, Sun W, Dong W, Wang Z, Zhang T, He L and Zhang H: Downregulation of long noncoding RNA H19 contributes to the proliferation and migration of papillary thyroid carcinoma. Gene 646: 98-105, 2018

25. Liu L, Yang J, Zhu X, Li D, Lv Z and Zhang X: Long noncoding RNA H19 competitively binds miR-17-5p to regulate YES1 expression in thyroid cancer. FEBS J 283: 2326-2339, 2016.

26. TamS,Boonsripitayanon M,Amit M,Fellman BM,LiY,BusaidyNL Cabanillas ME, Dadu R, Sherman S, Waguespack SG, et al: Survival in differentiated thyroid cancer: Comparing the AJCC cancer staging seventh and eighth editions. Thyroid 28: 1301-1310, 2018.

27. Ritchie ME, Phipson B, Wu D, Hu Y, Law CW, Shi W and Smyth GK: Limma powers differential expression analyses for RNA-sequencing and microarray studies. Nucleic Acids Res 43 : e47, 2015 .

28. Szklarczyk D, Franceschini A, Kuhn M, Simonovic M, Roth A, Minguez P, Doerks T, Stark M, Muller J, Bork P, et al: The STRING database in 2011: Functional interaction networks of proteins, globally integrated and scored. Nucleic Acids Res 39: D561-D568, 2011.

29. Smoot ME, Ono K, Ruscheinski J, Wang PL and Ideker T: Cytoscape 2.8: New features for data integration and network visualization. Bioinformatics 27: 431-432, 2011.

30. Gene Ontology Consortium: Gene ontology consortium: Going forward. Nucleic Acids Res 43: D1049-D1056, 2015.
31. Kanehisa M, Sato Y, Kawashima M,Furumichi M and Tanabe M: KEGG as a reference resource for gene and protein annotation. Nucleic Acids Res 44: D457-D462, 2016.

32. Dennis G Jr, Sherman BT, Hosack DA, Yang J, Gao W, Lane HC and Lempicki RA: DAVID: Database for annotation, visualization, and integrated discovery. Genome Biol 4: P3, 2003.

33. Ogluszka M, Orzechowska M, Jedroszka D, Witas P and Bednarek AK: Evaluate cutpoints: Adaptable continuous data distribution system for determining survival in Kaplan-Meier estimator. Comput Methods Programs Biomed 177: 133-139, 2019.

34. Kartsonaki C: Survival analysis. Diagnos Histopathol 22: 263-270, 2016.

35. Song B, Li R, Zuo Z, Tan J, Liu L, Ding D, Lu Y and Hou D: LncRNA ENST00000539653 acts as an oncogenic factor via MAPK signalling in papillary thyroid cancer. BMC Cancer 19: 297, 2019.

36. Xing M,Alzahrani AS, Carson KA, Viola D, Elisei R, Bendlova B, Yip L, Mian C, Vianello F, Tuttle RM, et al: Association between BRAF V600E mutation and mortality in patients with papillary thyroid cancer. JAMA 309: 1493-1501, 2013.

37. Moon S, Song YS, Kim YA, Lim JA, Cho SW, Moon JH, Hahn S, Park DJ and Park YJ: Effects of coexistent BRAF(V600E) and TERT promoter mutations on poor clinical outcomes in papillary thyroid cancer: A Meta-analysis. Thyroid 27: 651-660, 2017.

38. Fahiminiya S, de Kock L and Foulkes WD: Biologic and clinical perspectives on thyroid cancer. N Engl J Med 375: 2306-2307, 2016.

39. Xing M: Molecular pathogenesis and mechanisms of thyroid cancer. Nat Rev Cancer 13: 184-199, 2013.

40. Xie J, Fan Y and Zhang X: Molecular mechanisms in differentiated thyroid cancer. Front Biosci (Landmark Ed) 21: 119-129, 2016.

41. Sun Y, Liu WZ, Liu T, Feng X, Yang N and Zhou HF: Signaling pathway of MAPK/ERK in cell proliferation, differentiation, migration, senescence and apoptosis. J Recept Signal Transduct Res 35: 600-604, 2015.

42. Faustino A, Couto JP, Populo H, Rocha AS, Pardal F, Cameselle-Teijeiro JM, Lopes JM, Sobrinho-Simões M and Soares P: mTOR pathway overactivation in BRAF mutated papillary thyroid carcinoma. J Clin Endocrinol Metab 97: E1139-E1149, 2012.

43. Rusinek D, Swierniak M, Chmielik E, Kowal M, Kowalska M, Cyplinska R, Czarniecka A, Piglowski W, Korfanty J, Chekan M, et al: BRAFV600E-associated gene expression profile: Early changes in the transcriptome, based on a transgenic mouse model of papillary thyroid carcinoma. PLoS One 10: $\mathrm{e} 0143688,2015$.

This work is licensed under a Creative Commons Attribution-NonCommercial-NoDerivatives 4.0 International (CC BY-NC-ND 4.0) License. 